\title{
Assembly of Nanocrystalline Silicon Quantum Dots based on a Colloidal Solution Method
}

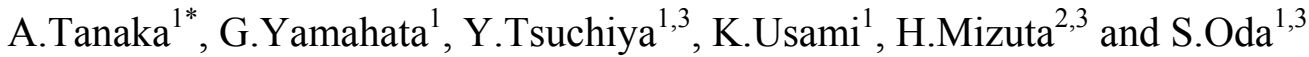 \\ ${ }^{1}$ Quantum Nanoelectronics Research Centre, Tokyo Institute of Technology, Tokyo, Japan \\ ${ }^{2}$ Department of Physical Electronics, Tokyo Institute of Technology, Tokyo, Japan \\ ${ }^{3}$ SORST, JST(Japan Science and Technology)
}

\begin{abstract}
In this paper we propose and develop a new bottom-up approach to the formation of silicon nanostructures based on assembly of nanocrystalline (nc) $\mathrm{Si}$ dots from the colloidal solution. The nc-Si dots with a diameter of $8 \pm 1 \mathrm{~nm}$ were fabricated by using VHF plasma decomposition of pulsed $\mathrm{SiH}_{4}$ gas supply and deposited on the substrate randomly. For preparing the nc-Si dot colloidal solution, we first examined various kinds of solvent. The substrates on which the nc-Si dots deposited were immersed into the solvents, and ultra sonic treatment was applied for a few tens seconds. It was found that methanol works as a suitable solvent for nc-Si dots. The nc-Si dot solution was then condensed by evaporating the solvent a fraction. We dropped the nc-Si dot solution onto other substrates and evaporated it completely. We observed that the nc-Si dots were assembled in the solution via the lateral capillary meniscus force which works as an attractive force between the dots. Use of $\mathrm{SiO}_{2}$ substrate with good surface wettability with the solution was found vital to have the maximum meniscus force and to have two-dimensional assembly of the dots. The evaporation speed was carefully controlled via temperature and evaporation pressure to achieve high dot density assembly. In addition, we examined the assembly of the nc-Si dots on the silicon-on-insulator substrates with various kinds of nanoscale patterning and succeeded in making the nc-Si dots cluster bridging between the nanoelectrodes with a gap of as small as $20 \mathrm{~nm}$.
\end{abstract}

Index Terms - nanocrystalline silicon, quantum dot, lateral capillary meniscus force, colloidal solution.

\section{INTRODUCTION}

Over the past few decades, the performance of siliconbased VLSI circuits has steadily been improved by scaling down device dimensions, and a nearly exponential growth of microelectronics capabilities has been achieved. However, maintaining this top-down miniaturization trend is getting exceedingly hard due to fundamental physical and technological limitations as well as of the economical limitation. Recently the bottom-up approach to form nanometer-scaled silicon structures with silicon nano-dots or nano-wires are attracting us more particularly for future nanoelectronics and quantum information device applications. Nanocrystalline silicon (nc-Si) is a promising material from a viewpoint of the integration with the silicon ULSI technology. We have succeeded in preparing an assembly of spherical nc-Si dots, with diameter of $8 \mathrm{~nm}$ and its dispersion less than $1 \mathrm{~nm}$ by using the VHF digital plasma process $[1,2]$. However, in the present deposition chamber, individual nc-Si dots are delivered by gas flow and deposited randomly on the substrates. Assembling the nc-Si dots over a large area on the substrate is very challenging issue. One potential application of the monodispersed nc-Si dot assembly is a planar cold electron emitter [3]. The cold electron emitting device was originally built by using porous silicon for a conducting region, and the electron transport along the nc$\mathrm{Si}$ dot chain structures plays an important role for reducing the energy loss of emitted electrons [4]. Therefore, a remarkable improvement is expected for the emission efficiency if an ordered array is introduced to the conducting region of the emitter. In addition, the nc-Si array formation technologies provide us with an enormous improvement of flexibility and reproducibility in device design and fabrication, in particular, for the single electron devices, the nano dot memory devices, and even for the future quantum information devices. Furthermore, various novel properties are expected to occur in such systems. For example, electrostatic interactions have been investigated for double $\mathrm{Si}$ dots [5] and for the twodimensional Si multidots [6]. Coherent wavefunction coupling and associated quasi-molecular states have also been observed for a tunnel-coupled double Si nanodots [7]. In addition, metal-insulator transition has been investigated for an artificial lattice of self-organized nanoparaticles [8].

Although our ultimate goal is to control the position of individual dots precisely, we currently focus on the twodimensional (2D) array formation as the first step. Techniques of fabricating a 2D ordered array of nanoparticles are roughly classified into the following three types; 1) direct manipulation after deposition, 2) deposition on the nano-patterned template and lift-off, and 3 ) the use of wet techniques in the field colloidal particles 
science. This first method is not suitable for future device integration because of its large time budget. In the second approach, although the recent development of nanotechnology facilitates synthesizing polymer templates with its pattern size down to several nanometers [9], further progress is still needed for applying them for the Si device processes. We therefore chose the third one, that is, the self-assembling of colloidal particle solved in a small quantity of liquid [10]. This method was intensively explored by Nagayama and his co-workers [11], and for example, ordering of ferritin macromolecules with the diameter of $12 \mathrm{~nm}$ was clearly observed [12]. In the previous studies of the formation of 2D array of nanoparticles, nanoparticle are usually synthesized in the liquid phase, whereas, our nc-Si spherical particles are grown in the gas phase. Therefore, preparing the nc-Si dot colloidal solution is an important issue in order for applying this technique to our nc-Si dot samples.

In this paper, we report on our recent progress of the solution drop \& evaporation technique [13] for making the nc-Si dot arrays. We investigate both the type of solvent and substrate suitable for assembling the nc-Si dots efficiently from the solution. Evaporation conditions are also studied to control the speed of the dot assembly. We study how our technique works both on flat and uniform substrates and on patterned substrates. We try to fabricate nanoscale transistors with the nc-Si dot cluster as a channel by apply the dot solution onto the SOI substrate with nanofabricated electrodes.

\section{EXPERIMENT}

Nanocrystalline Si dots with a diameter of $8 \pm 1 \mathrm{~nm}$ were deposited on the Si substrates in the UHV chamber by using VHF plasma decomposition of pulsed $\mathrm{SiH}_{4}$ gas supply. Area density of nc-Si dots is about $1 \times 10^{11} \mathrm{~cm}^{-2}$, corresponding to less than one monolayer in thickness. Immediately after the deposited substrate was taken out from the chamber (in about 10 seconds), we immersed it into a solvent and applied ultra sonic treatment to remove the nc-Si dots from the substrate surface and disperse them into the solvent. This process should be done before the surface of the nc-Si is fully oxidized and the strong bonding between the nc-Si dots and the substrate surface is formed. We examined three kinds of solvent: pure water, methano, 1 and cyclohexane in which oxidation is less likely to occur. After the ultra sonic treatment for about 5 minutes, we confirmed by using SEM that the nc-Si dots were removed from the Si wafer to large extent. We then take a drop of solution onto other clean $\mathrm{Si}$ substrate to observe if the nc-Si dots are indeed dispersed in the solution (see Fig.1).

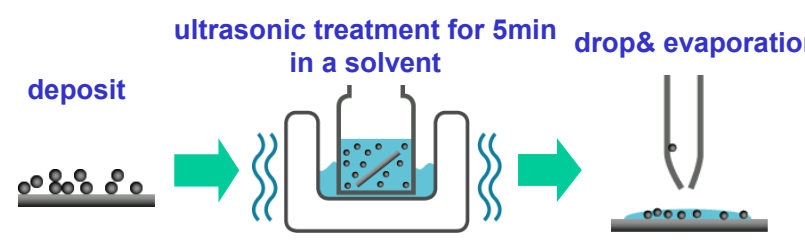

Fig. 1. A schematic flow-chart of the drop \& evapolation process.

Second we tried to use a drop and evaporation technique [13] to assemble the nc-Si dots from the dispersion solution. We utilized lateral capillary meniscus force [14] which works at the point where the three phases of the liquid, air and nc-Si dot meet (see Fig.2).

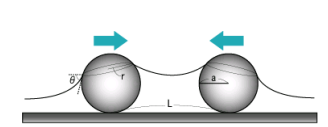

(a)

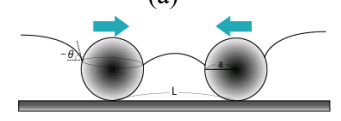

(c)

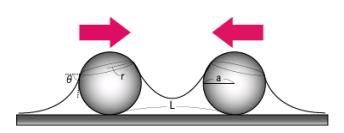

(b)

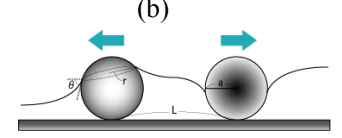

(d)
Fig. 2. Schematic illustrations showing how lateral capillary meniscus force works between two nc-Si dots. The dots receive the larger force when the level of solvent surface is lower and the dots have high wettability for the solvent. The dots receive attracting force when they have the same wettability.

So first we added a drop $(20 \mu \mathrm{l}$, and below all nc-Si

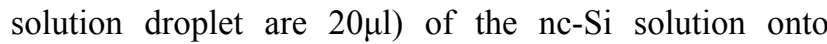
another Si substrate and evaporated the droplet at room temperature. We also applied it onto the $\mathrm{SiO}_{2}$ substrate because the surface of the substrate is hydrophilic and has good wettability for the solution. Therefore the level of the liquid surface is expected to be lower, and the lateral capillary meniscus force should work more efficiently. We also examined the effects of using isopropanol as a solvent as it has the higher boiling point of $82.2{ }^{\circ} \mathrm{C}$ compared with that of $64.5{ }^{\circ} \mathrm{C}$ for methanol. Evaporation of the solution was also performed at $-40^{\circ} \mathrm{C}$ to make the process slower.

Finally we applied the drop \& evaporation technique to the patterned SOI substrate. A heavily doped (n-type, phosphorous, doping concentration $\sim 1 \times 10^{20} \mathrm{~cm}^{-3}$ ) $50 \mathrm{~nm}-$ thick SOI wafer was prepared and nano electrodes were patterned by using an electron beam direct writing technique. Two terminal electrodes with a gap of about 30 $\mathrm{nm}$ have successfully been fabricated. This structure gives a good areal contrast of the surface wettability between 
the $\mathrm{Si}$ nanoelectrode regions and the $\mathrm{SiO}_{2}$ nanogap regions.

\section{RESULT AND DISCUSSION}

Figures 3 (a), (b) show the SEM images of the substrate before and after the ultra sonic treatment. We confirmed that the most nc-Si dots were removed from the substrate for three different solvents. This proves that the nc-Si dots are stuck onto the substrate due to the oxidation process within a few minutes after the sample is taken out from the UHV chamber. We then try to observe the nc-Si dots dispersed into the solvents by taking a drop of three solutions onto the other substrates. However, we could observe the nc-Si dot only for methanol. In the cause of pure water it is likely that nc-Si dots were etched like water marks [15] on Si wafer. Instead of the nc-Si dots we observed randomly shaped clusters as shown in Fig.4, which seem to be $\mathrm{Si}(\mathrm{OH})_{4}$. For cyclohexane we observed no change in the nc-Si dots left on the substrate, but we couldn't find any nc-Si dots from the solution. We believe that the nc-Si dots were not dispersed but aggregated in the solvent. This is probably because the solvent is composed of molecule with polarity, and the dots cannot have sufficient an electric repulsive force when covered with the molecules [16]. Therefore the nc-Si dots tend to stay attached once they touch each other and form large clusters stably. In summary only methanol can be used as solvent suitable for the nc-Si dots.

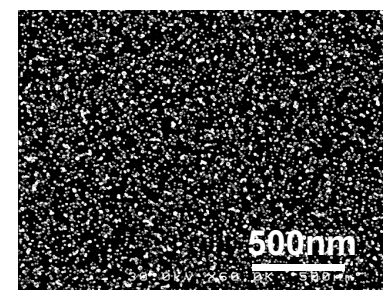

(a)

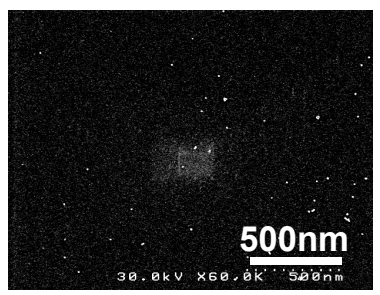

(b)
Fig. 3. SEM images of the nc-Si dots just after deposition (a) and after the ultra sonic treatment (b).

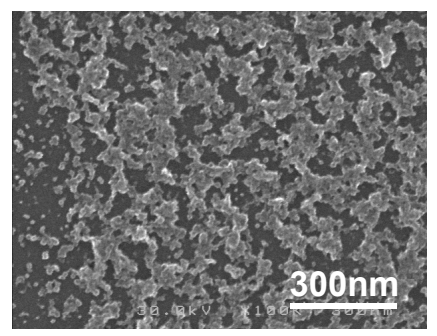

Fig. 4. A structure observed after the drop \& evaporation process with pure water.

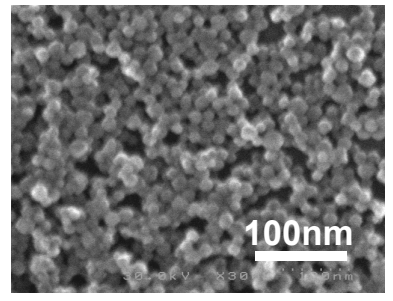

(a)

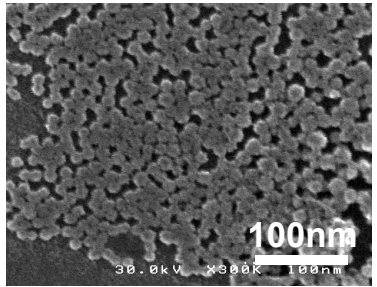

(b)
Fig. 5. SEM images of the nc-Si dots assembled at room temperature with the drop \& evaporation method using methanol solvent on $\mathrm{Si}(\mathrm{a})$ and $\mathrm{SiO}_{2}$ (b) substrates.

By using the prepared dispersion solution we then examined the drop \& evaporation method on both $\mathrm{Si}$ and $\mathrm{SiO}_{2}$ substrates. Evaporation of the solvent was done at room temperature and took about 40 minutes. Figure 5(a) shows the nc-Si dots assembled on the Si substrate. The nc-Si dots were assembled fairly randomly and stacked in multiple layers. On the other hand, the nc-Si dots on the $\mathrm{SiO}_{2}$ substrate got assembled in monolayer as shown in Fig. 5(b). Although there still remained lots of voids, the areal density of the nc-Si dots was found as high as $5 \times$ $10^{11} \mathrm{~cm}^{-2}$. For increasing the areal density further, the lateral capillary meniscus force should work among the nc-Si dots more effectively during the assembly process. One of the possible ways to do this is to make the assemble process slower. So we used isopropanol as a solvent in stead of

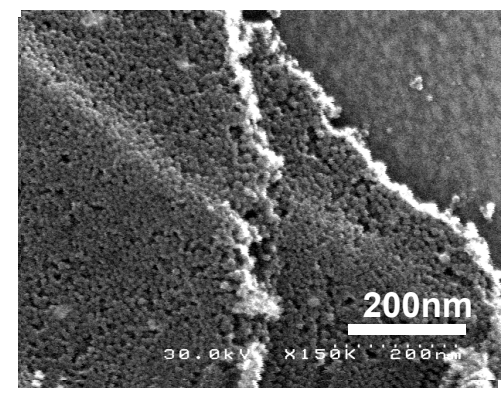

Fig. 6. The nc-Si dots assembled at $-40^{\circ} \mathrm{C}$ by the drop \& evaporation method using isopropanol solvent on the $\mathrm{SiO}_{2}$ substrate.

methanol and evaporated the droplet at $-40^{\circ} \mathrm{C}$, resulting in the evaporation time of about 24 hours. Figure 6 shows the nc-Si dots assembled under these conditions. The ncSi dots are densely packed with an areal density of about 
$9.4 \times 10^{11} \mathrm{dots} / \mathrm{cm}^{2}$. Over the large area it shows a layered structure with some steps. These results indicate that the lateral capillary meniscus force affected the nc-Si dots more strongly. The observed steps were probably formed when two (or multiple) assembled flat regions were pushed against each other by the lateral capillary force. The conditions on evaporation should be optimized for further improvement of the assembly process.

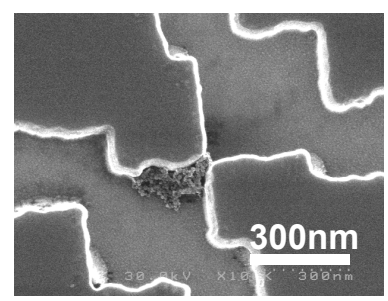

(a)

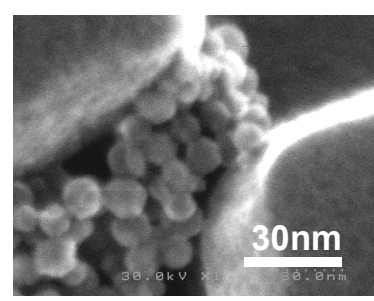

(b)
Fig. 7. SEM image of the nc-Si dots assembled on the SOI wafer with patterned nanoelectrodes (a) and the blow-up of the nanogap region (b).

Figures 7 (a), (b) show the nc-Si dots assembled on the SOI substrate with a patterned nanogap of about $30 \mathrm{~nm}$. This structure provides a regional contrast between hydrophobic ( $\mathrm{Si})$ and hydrophilic $\left(\mathrm{SiO}_{2}\right)$ surfaces. We observed that nc-Si dots remained only in the $\mathrm{SiO}_{2}$ regions and were assembled near the nano gap, resulting in a nc-Si dot channel between the electrodes. This trend was observed in common over the large area. This method may be useful to fabricate the nc-Si dot channel of nanoscale transistors, and we believe that it is possible to form a channel with few nc-Si dots or even a single dot by reducing the density of nc-Si and optimizing evaporation conditions.

\section{SUMMARY}

We developed the method of making the nc-Si dot dispersion solution and assembling the nc-Si dots from the solution by using the drop \& evaporation technique. Methanol was found to work best as a suitable solvent for nc-Si dots. The nc-Si dots were assembled successfully in the two-dimensional manner on the hydrophilic $\mathrm{SiO}_{2}$ surface via the lateral capillary meniscus force. By making the evaporation process slower at $-40{ }^{\circ} \mathrm{C}$ with use of isopropanol as a solvent, densely packed layered structures were formed with an areal dot density as high as $9.4 \times 10^{11} \mathrm{dots} / \mathrm{cm}^{2}$. In addition, we examined the assembly of the nc-Si dots on the patterned SOI substrates with nanoscale electrodes with a gap of as small as $20 \mathrm{~nm}$ and succeeded in fabricating the nc-Si dots cluster bridging between the electrodes. Combining the top-down nanolithography and bottom-up self-assembly, this technique may provide a new method to fabricate nanoscale Si structures for the future quantum information device applications.

\section{ACKNOWLEDGEMENT}

The authors wish to thank Dr Shin-ichi Saito and Dr Tadashi Arai of Central Research Laboratory, Hitachi Ltd. for many valuable discussions.

\section{REFERENCES}

[1] T. Ifuku, M. Otobe, A. Itoh, and S. Oda, Jpn. J. Appl. Phys. 36, 4031 (1997).

[2] K. Nishiguchi, S. Hara, and S. Oda., Mat. Res. Soc. Symp. Proc. 571, 43 (2000).

[3] K. Nishiguchi, X. Chao, and S. Oda, J. Appl. Phys. 92, 2748 (2002).

[4] Y. Nakajima, A. Kojima, and N. Koshida, Appl. Phys. Lett. 81, 2472 (2002).

[5] M. A. H. Khalafalla, H. Mizuta, and Z. A. K. Durrani, IEEE Trans. Nano-technol. 2, 271 (2003).

[6] R. Nuryadi, H. Ikeda, Y. Ishikawa, and M. Tabe, IEEE Trans. Nano-technol. 2, 231 (2003)

[7] M. A. H. Khalafalla, H. Mizuta, and Z. A. K. Durrani, Appl. Phys. Lett. 85, 2262 (2004)

[8] S. Saito, T. Arai, H. Fukuda, R. Tsuchiya, D. Hisamoto, S. Kimura, and T. Onai, International Conference on Solid State Devices and Materials, Tokyo, G-9-5 (2004)

[9] S. Oda, and K. Nishiguchi, J. Phys. IV France, 11, Pr3-1065 (2001).

[10] J. A. Theobald, N. S. Oxtoby, M. A. Phillips, N. R. Champness, and P. H. Beton, Nature 424, 1029 (2003).

[11] Y. Xia, B. Gates, Y. Yin, and Y. Lu, Adv. Mater. 12, $693(2000)$.

[12] N. D. Denkov, O. D. Velev, P. A. Kralchevsky, I. B. Ivanov, H. Yoshimura, and K. Nagayama, Nature 361, 26 (1993).

[13] Y. Tsuchiya, T. Iwasa, A. Tanaka, K. Usami, H. Mizuta, S. Oda, MRS Spring Meeting, April 2004.

[14] P.A.Kralchevsky, B.N.Paunov, N.D.Denkov, I.B.Ivanov and K.Nagayama, J.Colloid Interface Sci. 155,420 (1993).

[15] M.Watanabe, M.Hamano, M.Harazono, Materials Science and Engineering B4,(1989) p.4011

[16] H.Ohshima, Adv. Colloid Interface Sci., 53, 77 (1994). 